\title{
VALIDACIÓN DE UN MODELO ECONOMÉTRICO DE LETALIDAD POR INFECTADOS COVID-19, PERÚ MAYO 2020
}

\author{
LETHALITY ECONOMETRIC MODEL VALIDITY FOR COVID-19 INFECTED PLEOPLE, \\ PERU MAY 2020
}

\section{RESUMEN}

Objetivo: Probar mediante un modelo econométrico que la cantidad de los fallecidos en el Perú se relacionan de manera significativa con el número de casos infectados de COVID- 19. Método: Investigación de tipo básica, no experimental. Para todo el Perú se ha tomado 52 series (días) y en el caso específico del departamento de Moquegua, 37 series en el periodo del 16 de marzo al 10 de mayo del 2020. Se ha utilizado la base de datos del Ministerio de Salud, de la Sala situacional COVID-19 y de la Gerencia Regional de Salud de Moquegua; se emplea el R y $R^{2}$ de Pearson. Se generaron modelos de regresión el 10 de mayo (luego de 52 días del primer fallecido), que deben ser contrastados el 31 de mayo. Resultados: Los modelos cumplen con la predicción, con un $R^{2}$ y un Rho altos y significativos. Conclusiones: Los modelos de predicción de fallecidos son corroborados al 31 de mayo, luego de 73 días del primer fallecido en el Perú. Los niveles de correlación y determinación de Pearson en países que están saliendo de la emergencia del COVID-19, las regiones de todo el Perú tienen una alta y significativa relación entre infectados y fallecidos. A mayor número de infectados mayor número de fallecidos. En número y proporción son adultos y adultos mayores. En un $72,5 \%$ son varones. En Moquegua se demuestra que hay relación entre los niveles de exámenes diagnósticos realizados e infectados.

Palabras clave: Coronavirus; exámenes COVID-19; fallecidos; infectados; modelo econométrico.
Javier Pedro Flores Arocutipa Universidad Nacional de Moquegua Moquegua, Perú ORCID: https://orcid.org/0000-0003-0784-4153 Correo electrónico: javiefloresarocutipa@gmail.com

JORGE JiNCHUÑA HUALLPA Universidad Nacional de Moquegua Moquegua, Perú

ORCID: https://orcid.org/0000-0002-9073-3798 Correo electrónico: jjinchunah@unam.edu.pe

Roberto Tito Condori Perez Universidad Nacional de Moquegua Moquegua, Perú ORCID: https://orcid.org/0000-0001-6708-1675

Correo electrónico: rtcondorip@unam.edu.pe

[Recibido: 15/06/2020 Aceptado: 31/07/2020 Publicado: 31/08/2020]

Keywords: Coronavirus; COVID-19 tests; deceased; infected; econometric model. 


\section{INTRODUCCIÓN}

La enfermedad ocasionada por el coronavirus surgió en la ciudad china de Wuhan, capital de la provincia de $\mathrm{Hu}-$ bei, el pasado 8 de diciembre 2019. Lizaraso y Del Carmen (2020) refiere que, en el Perú, este virus fue detectado el 6 de marzo del 2020. Al 10 de mayo, cuando se debió levantar la cuarentena en el este país, había 1889 muertos y 65 mil contagios. En ese mismo periodo se podía observar en el portal web de la universidad de Johns Hopkins, contabilizaba 8.7 millones de exámenes diagnósticos realizados, cuatro millones de infectados y 281 mil muertes alrededor del mundo. También había 1.1 millones de recuperados. La tasa de letalidad era distinta por países; al 10 de mayo, por ejemplo, en Francia era del 18,67\%; en Alemania, 4,4\%; en Italia, 13,92\%; y en el Perú, 2,8\% (Johns Hopkins University, 2020).

En Lima, el 6 de marzo apareció el primer paciente infectado y luego se esparció a las demás regiones. La última región en detectar su primer infectado fue Ucayali, el 10 de abril.

En el diario Gestión (2020) y Perú 21 en la edición del 6 de marzo del 2020, se informó que el virus llegó al Perú luego que un hombre de 25 años visitara España, Francia y Republica Checa y otros países, además de otros viajeros del mundo que fueron infestados por el coronavirus COVID-19. Pero si bien llegó a los distritos de Jesús María, Miraflores, Surco, San Isidro, etc. donde viven familias de clase media la enfermedad pasó rápidamente a los distritos de mayor pobreza y hacinamiento como Villa María del triunfo, San Juan de Lurigancho, Villa el Salvador. Actualmente, las familias de los distritos en donde se evidencia fortaleza económica de lima están superando los niveles de infección, mientras que el virus aumenta en los distritos más pobres. Hoy, centros de investigación del país se interesan por desarrollar alternativas de vacuna contra el COVID-19.

Respecto a la pregunta de por qué se empecinan en crear una vacuna peruana cuando esta se está desarrollando en el primer mundo, la respuesta en cierta medida nos da la exministra de salud Patricia García: "Estoy en una comisión global para ver qué va a pasar con el acceso a la vacuna y, lamentablemente, parece que los países que ponen plata para la investigación pretenden tenerla primero. $\mathrm{Y}$ los países más chicos, que tenemos menos plata, menos casos, ¿ iseguiremos muriendo?” (Ampuero, 2020, párr. 7). Tal es así que Estados Unidos ya garantizó 100 millones de dosis con 1950 millones de dólares (ABC Sociedad, 2020) y países como Alemania, Francia, Italia y Holanda, 400 millones de dosis (Ortega, 2020). Entonces mucho tiene que ver la variable recursos para investigación en Salud y mayor presupuesto, para mejorar los servicios de salud por parte del Estado.

Estados Unidos tiene un Producto Bruto Interno (PBI) per cápita de 65 mil dólares y Alemania, de 50 mil; a diferencia de países como Turquía, Irán, Brasil y Perú que están por debajo de los 10 mil dólares de PBI per cápita. Como se puede ver, la relación es de uno a cinco entre países ricos y países emergentes. Perú tiene una relación de uno a nueve con Estados Unidos. Es por ello que, en el ranking del PBI per cápita, Estados Unidos se ubica en el séptimo puesto del mundo, mientras que Perú se ubica en el puesto 83 de 190 países. Tal vez eso explique que los países desarrollados enfrenten de mejor manera el COVID-19, para ello es importante observar que, hasta el 10 de mayo de 2020, el número de reproducción del virus " $R$ " en los países son los siguientes: USA (1); España (0.8); Italia (0.8); Reino Unido (1); Francia (0.6); Alemania (0.7) Brasil (1.4); Canadá (1.1); China (0). Hay que decir que en los países mencionados la tasa de contagio se duplica cada 10 días, Brasil cada 9 días y Perú peligrosamente todavía cada 8.3 días (Organización Panamericana de la Salud, 2019; The Global Economy, 2020; Saber es práctico, 2020; Population Pyramid, 2020).

El objetivo general de la investigación es demostrar que el número de fallecidos, producto de la enfermedad del Coronavirus, depende del número de infectados en el Perú, en el periodo del 06 de marzo al 10 de mayo del 2020, mediante nuestra validación econométrica.

Los objetivos específicos son: Demostrar que los niveles de crecimiento porcentual de fallecidos en el Perú es producto del incremento porcentual de infectados, para ello se plantea el modelo logarítmico. Asimismo, en el estudio se demostrará que el porcentaje mayor del número total de fallecidos en el Perú se explica por el número de adultos y adultos mayores. complementariamente se explicará mediante el método econométrico que los fallecidos en el Perú mayormente son del sexo masculino.

Las variables del estudio son las siguientes: Variable 1: Infectados; Variable 2: Fallecidos. Para ello se han planteado modelos econométricos y logarítmicos para el país y para la realidad de Moquegua.

Bayes, Sal y Rosas, y Valdivieso (2020) docentes de la Pontificia Universidad Católica del Perú presentaron un modelo de crecimiento y desarrollo del coronavirus en los contagiados y de manera decisiva sobre la cantidad de fallecidos. Este modelo fue elaborado el 8 de abril del 2020 y su objetivo era calcular el número de muertos debido al COVID-19, basado en los datos de muertes diarias en China, eligiendo el modelo predictivo lineal bayesiano. En sus principales conclusiones ellos afirman que el número 
de muertes que ocurrirán en el Perú sería de 612 fallecidos. Se afirmó que a los 64 días después de la primera muerte se observará el $99 \%$ de las muertes esperadas. Además, señalan que el punto de inflexión sería en el día 26 después de la primera muerte. La idea, como se aseveró, fue que las autoridades puedan monitorear la enfermedad y sobre todo implementar estrategias para contener la pandemia.

Se debe señalar que, en el Perú, en el día 26, después de la primera muerte había 181 fallecidos y al 10 de mayo, 1889. Pero el modelo hacía una comparación en el marco o modelo Wuhan que empezó el 8 de diciembre y terminó el 8 de marzo (noventa días). Otro de los trabajos es de Vincent Larivière, Fei Shu y Cassidy Sugimoto quienes resaltan las deficiencias en la comunicación científica por el brote del coronavirus. Es así que los autores se preguntan ¿hubo una asociación de intervenciones de salud pública con un mejor control del brote de COVID-19 en Wuhan, China? La pregunta surge por cuanto es la variable principal por responder (Lariviri, Shu y Sugimoto, 2020).

La enfermedad por COVID-19 se ha convertido en una pandemia, y se desconoce si una combinación de intervenciones de salud pública pueda mejorar el control del brote. Una serie de intervenciones de salud pública multifacéticas se asoció temporalmente con un mejor control del brote de COVID-19 en Wuhan, China. Estos hallazgos reflejan la política de salud pública en otros países y regiones para combatir esta pandemia mundial.

\section{MATERIALES Y MÉTODOS}

El estudio fue de tipo básica, no experimental. Se propone un modelo interpretativo y predictivo de la realidad para que, en el transcurso del 10 al 31 de mayo, se pueda notificar a los funcionarios de salud y del Gobierno Regional de Moquegua y sirva, tal vez, para modificar la realidad.

En cuanto a la muestra, para los modelos nacionales se contabilizó desde el 6 de marzo al 10 de mayo, que son 65 días o series. La propuesta es hacer el seguimiento hasta el 31 de mayo o hasta que se llegue al final de la serie por disminución de infectados y fallecidos; en total son 87 días de análisis.

En cuanto al cálculo de los modelos para hacer las predicciones se utilizó el programa Eviews 10, previo de hacer la recopilación en el programa Excel de Microsoft. Se utilizaron los estadísticos conocidos como el coeficiente de correlación de Pearson para datos cuantitativos; el coeficiente de determinación de Pearson para calcular los modelos causales, explicativos; y el software Eviews para modelos logarítmicos o de impacto.

\section{RESULTADOS}

Aplicando el coeficiente de correlación de variables en el Perú, se obtiene que la variable fallecidos se relaciona con la variable infectados $(0,999)$, con el tamaño de muestra y con los hospitalizados. También hay una relación alta y significativa entre las muestras tomadas y el número de infecciones $(0,997)$. Entonces el nivel de muertes tiene correlación alta y significativa con la variable infectados $(0,999)$ y con hospitalizados $(0,986)$. La única variable manipulable en su aplicación sería la variable muestras dado que dependerá si existen test en stock y si hay la voluntad de realizar los mismos.

El modelo encontrado tiene un $\mathrm{R}^{2}$ de 0,9990 que es bastante significativo, los betas cumplen el requisito que su probabilístico sea menor que 0,05 , y que el probabilístico de F sea menor que 0,05 lo cual no indica que el modelo como conjunto es adecuado.

$$
\begin{aligned}
\text { Muertes } & =6,75257777485+0,037846755662 * \\
& \text { INFECTADOS }-0,00203574200158 * \\
& \text { MUESTRAS }+0,0558710219654^{*} \text { HOSPITAL }
\end{aligned}
$$

$\mathrm{R} 2=0.9990$, betas: bo $(0.1025) \mathrm{B} 1(0.0000) \mathrm{B} 2(0.0000) \mathrm{B} 3$ (0.000); Prob F (0.00000)

a. Letalidad, número de fallecidos en el Perú. Si bien al empezar la pandemia del COVID-19 en el Perú, la preocupación era el número de infectados, esta se incrementó cuando se empezó a contabilizar el número de infectados en Lima, en Loreto, en Callao y en Lambayeque. Las pruebas rápidas hicieron crecer las muestras y al haber infectados, estos eran detectados. $\mathrm{Al}$ respecto la correlación entre muestras e infección era de $99,77 \%$, alta y significativa. Y el coeficiente de determinación de Pearson fue de 99,54\%, esto quiere decir que a más muestras habrá, definitivamente, mayor cantidad de infectados y que el tamaño de muestras sí explica el nivel de infectados de manera significativa. Pero hoy el problema es la letalidad. En ese sentido, desde el 5 de abril de 2020 la tasa de infectados respecto de la muestra es en promedio el $13 \%$. Quiere decir que, de cada 100 muestras, 13 resultan infectados. Una vez ubicados los infectados se debe saber el nivel de letalidad, luego que algunos pasaran por hospitalización y otros que llegaron a camas UCI, se debe calcular qué proporción de pacientes resultaron muertos. Lo cierto es que la tasa de letalidad promedio en el Perú, para el 28 de mayo fue de 2.89 del 27 de abril que fue de 2,72\%.

También se debe decir que la correlación entre el número de infectados y el número de muertos es de $99,9 \%$, muy significativa y alta. Quiere decir que el número de infectados corre en paralelo con el número de fallecidos. Se 
afirma que el número de infectados sí explica el número de muertos en $99,75 \%$. Quiere decir que hay certeza que de cada 100 infectados, hay 3 fallecidos. Por ello a nivel nacional, al 10 de mayo, hay 65425 infectados y son 1889 los fallecidos.

\section{Los adultos mayores}

La tasa de letalidad es de $2,81 \%$ en promedio y se concentra en los adultos mayores. De los 1889 muertos, los fallecidos adultos mayores son 1264, que representa el $67 \%$. Los adultos fallecidos fueron 599, que representa el 31,6\%. Los jóvenes fallecidos son 16; los adolescentes, 3 ; y los niños, 7. Como se puede notar, entre adultos y adultos mayores, son el $98,6 \%$ del total de fallecidos. ¿Y cuántos infectados son necesarios para que aparezcan los fallecidos? Es poco predecible. A nivel nacional, hubo 117 infectados cuando falleció el primer peruano. Tacna, con 128 infectados, tuvo dos fallecidos; Huancavelica con 33 infectados, ya tenía un fallecido; San Martin, con 121 infectados, registró el primer fallecido; Pasco, con 48 infectados, tuvo un fallecido; Huánuco, con 160 infectados, registró un fallecido; Cajamarca, con 52 infectados, ya tenía 2 fallecidos. Se concluye que, con un promedio de 100 infectados, se tiene un fallecido; aunque la relación ha ido disminuyendo conforme transcurren los días.
Otro de los aspectos por considerar es que entre los que fallecen mayormente son varones que mujeres. Así, de las 1889 personas fallecidas al diez de mayo, 1356 son varones y 533 son mujeres.

Se observa que, en términos porcentuales, el $71,8 \%$ son varones y el $28,2 \%$ son mujeres. De hecho, se señala que fallecen hasta dos veces más hombres que mujeres (Ver Figura 1).

Desde el 25 de abril hasta el 10 de mayo, los últimos dieciséis días, en el país el número de fallecidos se incrementó de 700 a 1889 fallecidos, 1189 muertos más. Es decir 79 muertes diarias, pero la media del 5 al 10 de mayo era de 86 fallecidos.

Al diez de mayo del 2020, solo había fallecido el 1,4\% de las personas en edad de 0 a 29 años. El mayor porcentaje de letalidad se concentraba en los grupos de adultos y adultos mayores con un $98,6 \%$ del total. En los últimos 16 días, el fallecimiento se ha incrementado en adultos mayores: 806 personas. En adultos el incremento ha sido de 369; mientras que en jóvenes el incremento es de 9; en niños, 3 ; y en adolescentes el incremento ha sido de 2 fallecidos. Se puede observar que el efecto es mayor en los adultos y adultos mayores. Otra forma de observar el fallecimiento

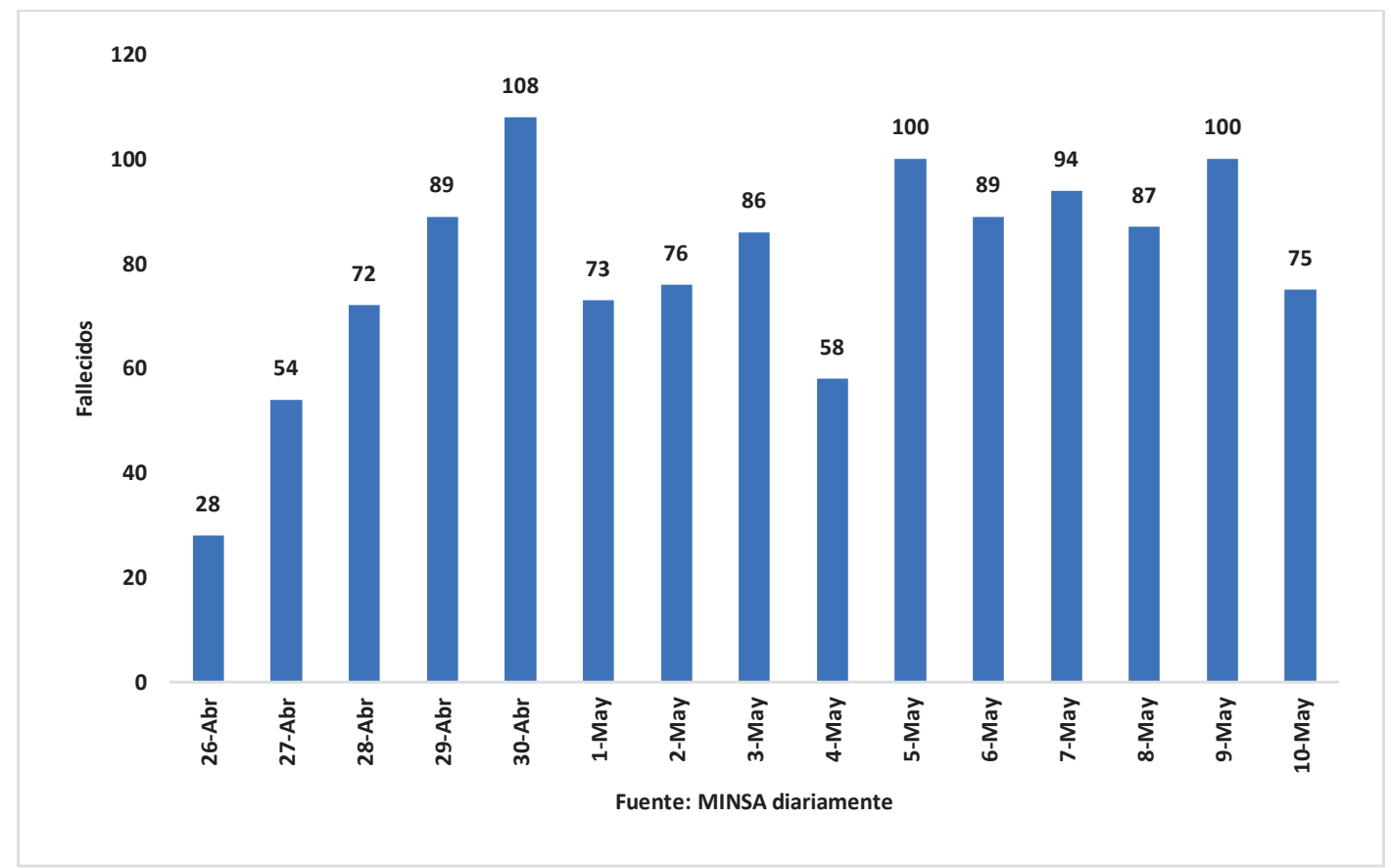

Figura 1. Gráfico del promedio de fallecidos por día son 79. El rango va de 80 hasta el día 30 de abril. Luego el rango es de 50 días.

Fuente: Elaboración propia con información publicada por el Ministerio de Salud. 
al 10 de mayo es por segmentos de edad. Así, de 1889 personas, de 0 a 29 años, han fallecido 26 personas; y de 0 a 39 años, 91 personas. Si agregamos a las personas de 40 a 49 años sumarán con los anteriores la cifra de 242 personas, que es el $12,8 \%$. Se colige que el porcentaje mayor está concentrado en las edades de 50 a más años, con el 87,2\%.

\section{Modelos para Perú}

Entonces es preciso demostrar que, efectivamente, el número de fallecidos depende del número de infectados, de tal manera que el coeficiente de determinación es de 0,9937 o $99,37 \%$.

1. Modelo calculado el 10 de mayo:

$$
\text { Muertes }=-11,44+0,028 * \text { Infectados }
$$

R2=0,998090; betas Bo (0,0000); DW (0,383105); Prob F $(0,0000)$

El modelo de impacto es:

$$
\log (\text { muertes })=-4,5834+1,0993 * \log (\text { infectados })
$$

Si se incrementa en uno por ciento los infectados, el número de fallecidos se incrementa en 1,099\%

2. El modelo econométrico del total de fallecidos, respecto de adultos y adultos mayores es:

$$
\begin{gathered}
\text { Muertes }=0,69184+0,9833 * \text { adulto mayor }+1,0772 \\
* \text { adulto }
\end{gathered}
$$

Con un DW de 0,965 ; con un F prob $(0,000)$; y betas $(0,4775)(0,000)(0,000)$.

Donde el $\mathrm{R}^{2}$ es de $99,99 \%$ y el R ajustado es de $99,99 \%$. Es un modelo altamente significativo.

Si se trata del impacto, la totalidad de muertos se explica por los fallecidos adultos y adultos mayores en el Perú. Siendo mínimo la afectación a las personas jóvenes, adolescentes y niños. $\mathrm{Al}$ respecto se ha encontrado el modelo siguiente:

$$
\begin{aligned}
\log (\text { muertes })= & 0,67320273+0,64708 * \log (\text { adultos } \\
& \text { mayores })+0,3516 * \log (\text { adultos })
\end{aligned}
$$

Significa que el incremento de $1 \%$ de fallecidos adultos mayores, incide en el total de fallecidos en $0,64 \%$. De la misma manera, el incremento del $1 \%$ de fallecidos adultos incide en un $0,35 \%$ en el total de fallecidos. El impacto mayor es con el incremento de fallecidos adultos mayores. El modelo tiene un $\mathrm{R}^{2}$ de $99,99 \%$ y un probabilístico $\mathrm{F}$ de $(0,000)$. Asimismo, la probabilidad de los coeficientes es de 0,0000 .
De la misma manera, se genera el modelo por edades y fallecidos; para ello cada 9 años es un segmento. Así las edades iban de 0-9; 10-19; 20-29, 30-39; 40-49; 50-59; 60-69; 70-79; 80 a más. Y el 10 de mayo se encontró el siguiente modelo:

$$
\begin{aligned}
\text { Muertes } & =46,4259777627+1,63869672767 \\
& *(70-79)+1,36199177791 *(50-59)+ \\
& 1,14434909922 *(60-69)
\end{aligned}
$$

Los resultados al 10 de mayo fueron de 1894 muertes cuando el histórico fue 1889 , una diferencia de -5 fallecidos, un margen $0,28 \%$ que es aceptable.

\section{Correlato de fallecidos por sexo}

3. Los que mayormente fallecen en el Perú (al 10 de mayo) son varones en un promedio de $71,8 \%$ y mujeres en un $28,2 \%$. Este es un primer dato y para ello se ha detectado que la correlación entre el total de fallecidos y varones es de 99,99\% y entre fallecidos y mujeres es $99,95 \%$.

El modelo que hemos encontrado es:

\section{Muertes=-3,88298808543+1,39135234137*Masculino}

Con un $\mathrm{R}^{2}=0,999850$; betas $(0,3812)$; $(0,0000)$; Dw $(0,7398)$; $\mathrm{F}$ prob $(0,000)$. Un modelo altamente significativo.

Y si hablamos de impacto del sexo, la idea es demostrar que los fallecidos en el Perú mayormente son del sexo masculino al 10 de mayo del 2020.

Modelo logarítmico:

$$
\begin{aligned}
\log (\text { muertes }) & =0,329690453046+ \\
& 0,999615435675^{*} \text { LOG (Masculino) }
\end{aligned}
$$

$\mathrm{R} 2=0,999817 ;(0,00000) ;(0,0000) ; \mathrm{DW}(0,7732) ; \mathrm{F}$ (prob) 0,0000

Se colige que a un incremento del $1 \%$ de fallecidos varones el incremento en el total de fallecidos es del 0,99\%, y si el incremento es del 10\% de varones fallecidos el incremento en el total es de 9,9\% (Ver Figura 2).

\section{Moquegua}

El número de infectados en la región Moquegua depende de manera significativa de las muestras tomadas en el periodo del 4 abril al 10 de mayo del 2020.

Los niveles de correlación de Pearson son de 99,45\% que es bastante alto y significativo. En cuanto a los niveles de explicación es de 98,90\%; en ese sentido es también significativo. El modelo es: 


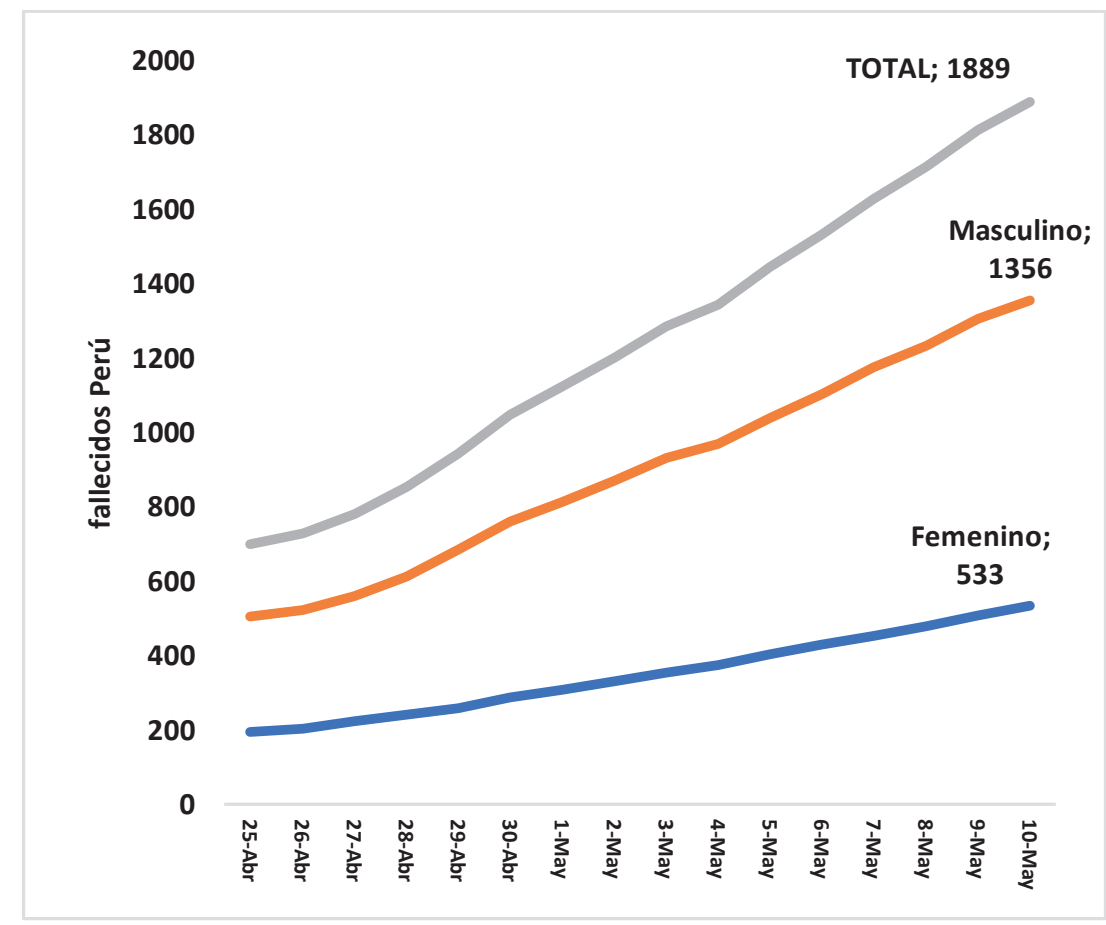

Figura 2. Gráfico de varones que fallecen por efecto del COVID-19 en mayor proporción que las damas. Al 10 de mayo hay 1889 muertos.

Fuente: Elaboración propia con información publicada por el Ministerio de Salud.

\section{Infectados $=-3,39296280547+0,0366935047927 *$ Muestra}

Con un $\mathrm{R} 2=0,989$; con un $\mathrm{F}$ (prob) $(0,000)$, con betas Bo $(0,040)$ B1 $(0,000)$.

En cuanto al impacto tenemos el modelo logarítmico para Moquegua es el siguiente:

$$
\begin{aligned}
& \log (\text { Infectados })=-5,07829257385+ \\
& 1,2215887515^{*} \text { LOG (Muestra) }
\end{aligned}
$$

El cual señala que a un incremento de $1 \%$ de muestras el impacto en infectados es de $1,22 \%$. Si las muestras o exámenes diagnósticos realizados se incrementan en $10 \%$ el incremento de los infectados es de $12,2 \%$.

\section{Contrastación de los modelos al 31 de mayo}

1. El coeficiente de correlación de Pearson para Perú al 31 de mayo era de 0,9994 entre fallecidos e infectados y el coeficiente de determinación de Pearson $\mathrm{R}^{2}=$ 0,999; quiere decir que el nivel de infectados explica el nivel de fallecidos. Al 31 de mayo, el número de infectados era de 164 476; y el de fallecidos, de 4505. Al aplicar el modelo encontrado, al 31 de mayo el número de infectados era de 164 476; y los fallecidos,
4600, una diferencia de 95 , lo cual porcentualmente significa $2,6 \%$.

2. Al aplicar el modelo de fallecidos que dependen de los grupos etéreos adultos mayores y adultos, el 10 de mayo se encontró 1889 fallecidos que fueron los resultados acumulados de ese día con 1264 fallecidos adultos mayores y 599 fallecidos adultos. El 31 de mayo, aplicando el modelo encontramos $4499 \mathrm{fa}-$ llecidos, una diferencia de 7 fallecidos, dado que los resultados históricos fueron de 4506, una diferencia en porcentaje de $0,16 \%$. Asimismo, al 31 de mayo nuestro modelo de regresión por edades generó 4450 muertes, mientras que el histórico fue de 4506 fallecidos, una diferencia de 56 muertes (1,25\%). Al 31 de mayo, los fallecidos de 70 a 79 años fueron 1087; de 50 a 59, 841; y de 60 a 69 años, 1291 fallecidos.

3. Respecto a los fallecidos que dependen del sexo, aplicado el modelo al 10 de mayo los resultados fueron de 1883 fallecidos, cuando el histórico fue de 1889 muertos. Una diferencia de 6, que representa el 0,32\%. Entretanto el 31 de mayo los resultados con el modelo fueron de 4492 fallecidos, mientras que el histórico fue de 4506 . Una diferencia de 14 , que es el $0,32 \%$. 


\section{Proyecciones para la Región Moquegua}

Al 31 de mayo, con el modelo calculado el 10 de mayo, hubo 606 infectados. El histórico fue de 508. Una diferencia de 98 infectados (variación del 16\%). Diferencia significativa que explica por qué en los primeros 37 días se realizaron 4260 exámenes diagnósticos y entre el 11 al 31 de mayo (21 días) se realizaron 12336 exámenes diagnósticos. Dos veces más en menos días. Al 31 de mayo se habían realizado 16596 exámenes.

\section{DISCUSIÓN}

En otros estudios, como series publicadas en Nueva York, se encontró que la edad promedio de letalidad era de 63 años; y el 60,3\% de los afectados era hombres. Asimismo, en una investigación realizada en Italia, se encontró resultados similares, presentándose más en varones. (Richardson, Hirsch y Narasimhan, 2020; Onder, Rezza y Brusaferro, 2020). Se concluye que, en el Perú, al 31 de mayo se corrobora que hay fuerte relación entre infectados y fallecidos; a mayor número de infectados mayor número de fallecidos, tal cual ocurrió en países en los cuales la tasa de infección se encuentra reduciéndose. Los fallecidos en su mayoría son adultos mayores, adultos y varones.

\section{REFERENCIAS}

Ampuero, A. (21 de mayo de 2020). Vacunas: el Perú está lejos de dar con una y ensaya tratamiento con gallinas. La República. Recuperado de: https://larepublica. pe/sociedad/2020/05/21/coronavirus-en-peru-vacunas-el-pais-esta-lejos-de-dar-con-una-y-ensaya-tratamiento-con-gallinas/

Bayes, C., Sal y Rosas, G., \& Valdivieso, L. (2020). Modelling death rates due to COVID-19: A Bayesian approach. Lima: Pontificia Universidad Católica del Perú. Recuperado de https://arxiv.org/pdf/2004.02386.pdf

ABC Sociedad. (24 de junio de 2020). Estados Unidos compra 100 millones de dosis de la posible vacuna contra el coronavirus a Pfizer y BioNTech. Diario ABC. Recuperado el 2 de Agosto de 2020, de https:// www.abc.es/sociedad/abci-estados-unidos-compra-100-millones-dosis-posible-vacuna-contra-coronavirus-pfizer-y-biontech-202007231143_noticia.html?ref=https:\%2F\%2Fwww.google.com\%2F

Ortega, E. (13 de Junio de 2020). Italia, Francia, Holanda y Alemania compran 400 millones de dosis de una vacuna sin testar del Covid-19. El Español. Recuperado de: https://www.elespanol.com/invertia/observatorios/sanidad/20200613/alemania-italia-francia-holanda-millones-no-astrazeneca/497450721_0.html
Investigadores peruanos trabajan en posible vacuna contra el Covid-19. (12 de abril de 2020). Gestión. Recuperado de: https://gestion.pe/peru/coronavirus-peru-investigadores-trabajan-en-posible-vacuna-contra-el-covid-19-nndc-noticia/

Johns Hopkins University \& Medicine. (2020a). Coronavirus. Resource Center. Recuperado de: https://coronavirus.jhu.edu

Johns Hopkins University \& Medicine. (2020b). COVID-19 Dashboard by the Center for Systems Science and Engineering (CSSE) at Johns Hopkins University. Recuperado de: https://coronavirus.jhu.edu/map.html

Larivière, V., Shu, F., \& Sugimoto, C. (12 de marzo de 2020). El brote de coronavirus (COVID-19) resalta serias deficiencias en la comunicación científica [mensaje en blog]. Recuperado de: https://blog.scielo.org/ es/2020/03/12/el-brote-de-coronavirus-covid-19-resalta-serias-deficiencias-en-la-comunicacion-cientifi$\mathrm{ca} / \# . X r j I C m h K i H s$

Lizaraso, F. y Del Carmen, J. C. (2020). Coronavirus y las amenazas a la salud mundial. Horizonte Médico, 20(1), 4-5. . doi.org/10.24265/horizmed.2020.v20n1.01

Onder, G., Rezza, G., y Brusaferro, S. (2020). Case-Fatality Rate and Characteristics of Patients Dying in Relation to COVID-19 in Italy. Jama, 323(18), 1775-1776. doi. org/10.1001/jama.2020.4683

Organización Panamericana de la Salud. (2019). Indicadores Básicos 2019: Tendencias de la Salud en las Américas. Recuperado de https://www.paho.org/data/ index.php/es/indicadores.html

Population Pyramid. (2020). Population Pyramids of the World from 1950 to 2100. Recuperado de https://www. populationpyramid.net/

Richardson, S., Hirsch, J., y Narasimhan, M. (2020). Presenting Characteristics, Comorbidities, and Outcomes Among 5700 Patients Hospitalized With COVID-19 in the New York City Area. Jama, 323(20), 2052-2059. doi:10.1001/jama.2020.6775

Saber es práctico. (2020). Población de los departamentos del Perú. Recuperado de https://www.saberespractico. $\mathrm{com} /$ ?s=Peru

The Global Economy. (2020). Crecimiento económico, clasificaciones. Recuperado de: https://es.theglobaleconomy.com/rankings/Economic_growth/ 
